\title{
Pancreatic Schwannoma Presenting as Gastric Outlet Obstruction- A Rare Presentation
}

\author{
Kumar Jayant ${ }^{1, *}$, Swati Agrawal ${ }^{2}$, Rajendra Agarwal $^{3}$ \\ ${ }^{1}$ Department of Surgery, Sudha Hospital \&Medical Research Centre, Talwandi, Kota, Rajasthan, India \\ ${ }^{2}$ Sudha Hospital \& Medical Research Centre Kota, Rajasthan, India \\ ${ }^{3}$ Department of Surgery, Sudha Hospital \&Medical Research Centre Kota, Rajasthan, India \\ *Corresponding author: jayantsun@yahoo.co.in
}

Received December, 21, 2013; Revised December 28, 2013; Accepted January 16, 2014

\begin{abstract}
Introduction: Schwannoma is a very uncommon pancreatic neoplasms. Because of their varing size, cystic nature they mimic pancreatic cystic neoplasms. Mostly definitive diagnosis is confirmed with histological assessment post resection. The mainstay of treatment is surgical resection based on the nature of tumour. Case presentation: Here we are reporting a very rare case of pancreatic schwannoma in 45 year old female presenting with pain upper abdomen with projectile vomiting. Pre-operative diagnosis of heterogenous tumor arising from head of pancreas was made on CECT abdomen following which Whipple's procedure was performed. Post operative histological analysis showed spindle cells arranged in palisading pattern with S-100 positivity, confirms the diagnosis of pancreatic schwannoma. Conclusion: We conclude that pancreatic schwannoma must be considered as an important differential diagnosis of pancreatic neoplasms. Though they have non specific symptoms but can present in unusual way as gastric outlet obstruction. Pre-operative diagnosis is difficult though computed tomographic and magnetic resonance imaging may be of value. For benign tumors, simple enucleation is enough, while malignant tumors requires complete oncological resection.
\end{abstract}

Keywords: pancreatic tumor, pancreatic schwannoma, schwannoma

Cite This Article: Kumar Jayant, Swati Agrawal, and Rajendra Agarwal, "Pancreatic Schwannoma Presenting as Gastric Outlet Obstruction- A Rare Presentation.” American Journal of Clinical Medicine Research 2, no. 1 (2014): 4-7 doi: 10.12691/ajcmr-2-1-2.

\section{Introduction}

Schwannomas are very rare neoplasms, also known as neurilemmomas and typically arise in the extremities, while other important locations are trunk, head and neck, pelvis, and rectum [1]. 60\% tumors are benign compared to $10 \%-15 \%$ are malignant. Pancreatic schwannomas are of neurogenic origin tumour arising from sympathetic or parasympathetic fibers. Pancreas is an one of very uncommon location for schwannomas and predominantly seen in adults, with an equal sex distribution [2]. We report a rare case of pancreatic schwannoma localized in head and uncinate process, presenting with features of pain upper abdomen with gastric outlet obstruction. This is a very rare presentation of this tumour not reported in literature so far.

\section{Case Presentation}

A 45-year-old female presented at our outpatient department with a history of intermittent upper abdomen pain and fullness with projectile vomiting occurring 12-16 hours after last meal suggestive of gastric outlet obstruction. Pain was of moderate intensity, non radiating and was associated with weight loss. On physical examination her abdomen was soft, fullness in upper abdomen with no palpable mass, but mildly tender to palpation in the epigastrium. On laboratory tests, as hemoglobin(10 g/dl), LFT, RFT and chest X-ray were within normal limit along with tumor markers as carbohydrate antigen (CA) 19-9 and carcinoembryonic antigen (CEA) were normal.

A contrast computed tomographic (CT) scan demonstrated a $45 \times 55 \mathrm{~mm}$ mass in the pancreatic head and uncinate process compressing duodenal lumen partially. (Figure 1) Based on these finding she was taken whipple's procedure. Laparotomy showed $6.5 \mathrm{X} 6 \mathrm{~cm}$ hard growth in body and uncinate process, abutting SMA with partially compromised duodenal lumen, while CBD and PD not dilated. No ascites, lymphadenopathy, liver, omental, or peritoneal mets seen. Cut Section shows growth in uncinate process with central necrosis. (Figure 2) Specimen was sent for histological analysis showed a schwannoma with spindle cells arranged in a palisading pattern. (Figure 3) Immunohistochemistry showed S100 positive tumor cells. Postoperative period was uneventful, and on further follow ups till 3 years she remained symptom free. 


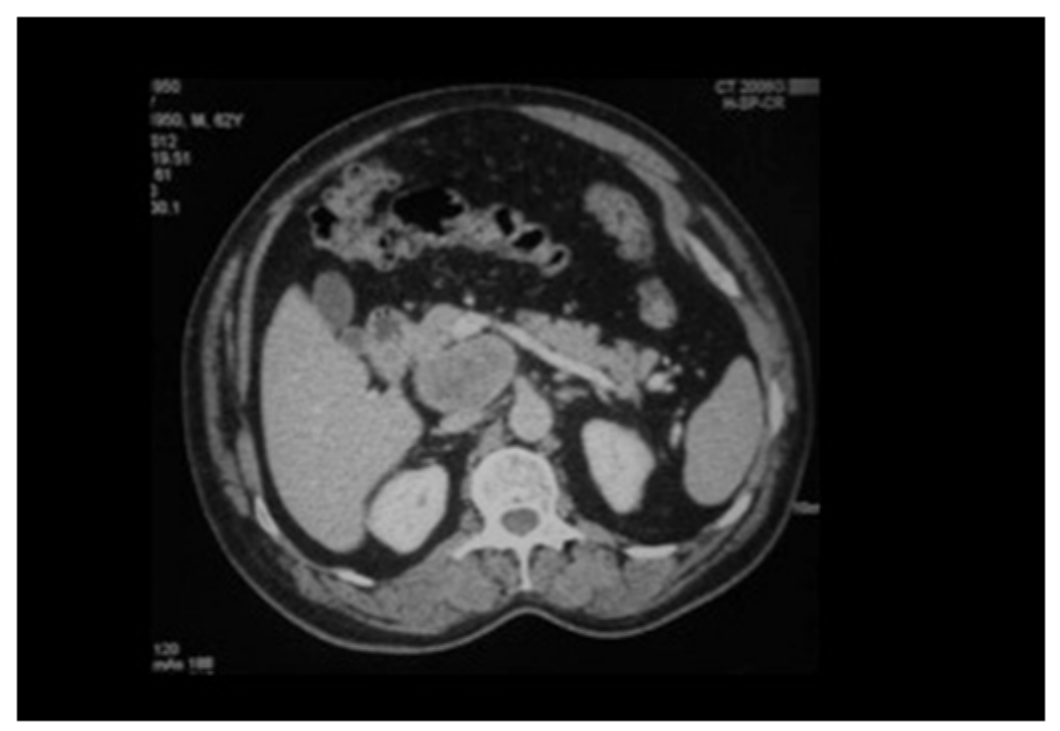

Figure 1. Computed Tomographic (CT) scan demonstrated a $45 \times 55 \mathrm{~mm}$ mass in the pancreatic head and uncinate process duodenal lumen compromise

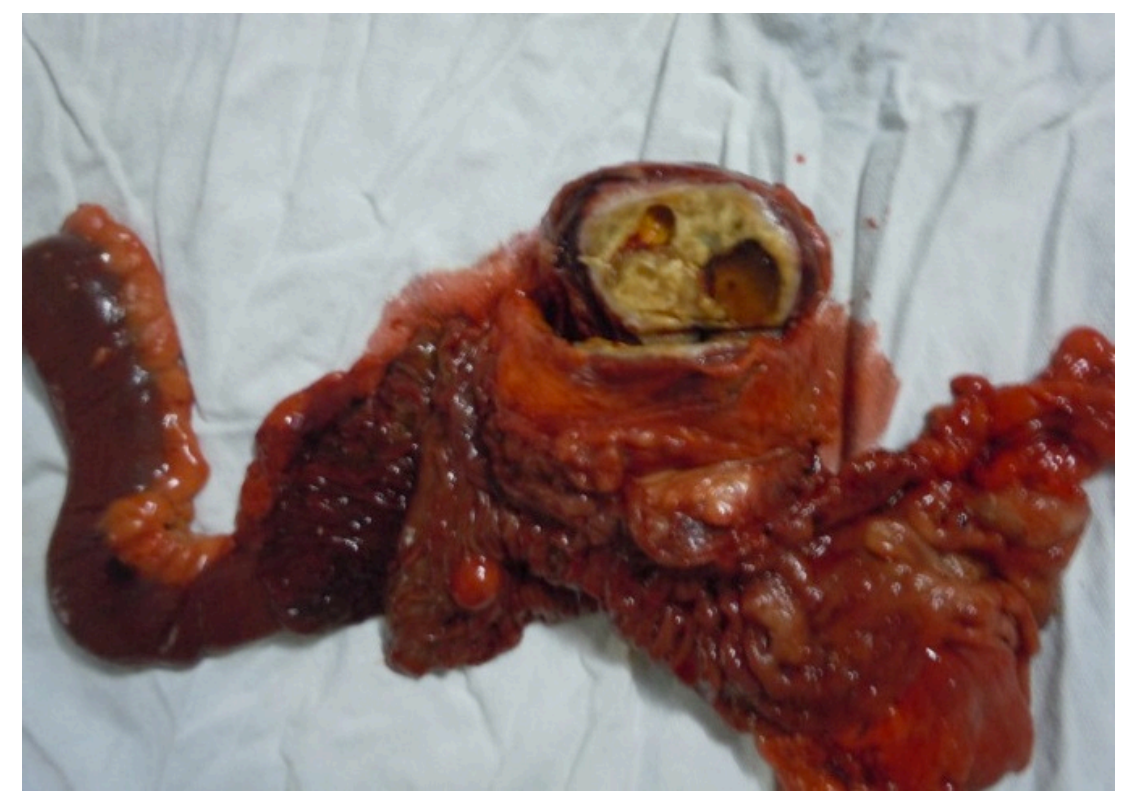

Figure 2. $6.5 \mathrm{X} 6 \mathrm{~cm}$ hard growth in body and uncinate process, abutting SMA with compromised duodenal lumen

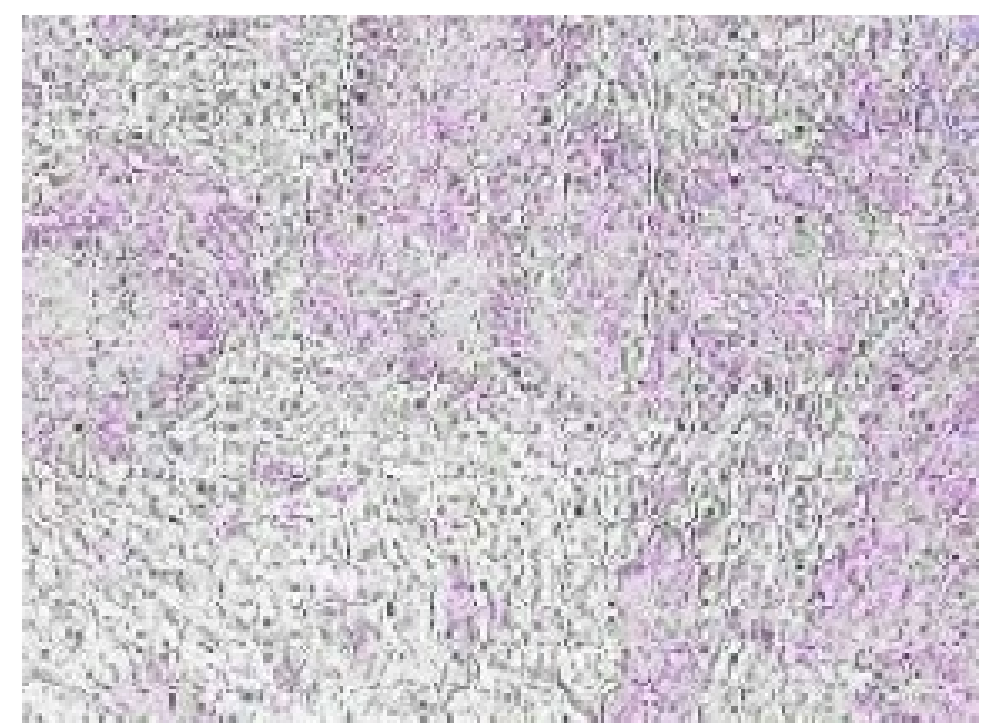

Figure 3. Microscopy showed spindle cells arranged in a palisading pattern 


\section{Discussion}

Even extensive search of literature related with pancreatic schwannoma we found less than 30 cases. Most of these neoplasm are of varing sizes $(2-22 \mathrm{~cm})$ and mainly found in the head (40\%) and body (22\%) region of the pancreas.[3] They are slow growing tumor of neurogenic origin arising from either sympathetic or parasympathetic fibers, which embryologically runs within pancreas with vagus nerve. More than half of them are cystic and $80 \%$ are benign.[4] Malignant form pancreatic schwannomas are also seen, though rare and till date only four cases have been reported so far. Malignant transformation is seen in $8 \%$ of cases in association with von Recklinghausen's disease. Predominant symptoms are nonspecific abdominal pain with weight loss, jaundice, and gastrointestinal bleeding though our case was unique because none of patient presented with features of gastric outlet obstruction [5].

CT scan is a primary investigation in evaluation and the most important finding is well defined loci of low density with few cystic areas which are either the Antoni B component or cystic degeneration of the schwannoma. Some times such degeneration create confusion in diagnosis because of resemblance with cystic neoplasm of pancreas. Contrast-enhanced CT is usefull in differentiating between Antoni A and Antoni B areas based on their vascularity as enhanced regions closely match with Antoni A, and unenhanced regions concur with Antoni B. [6] Magnetic resonance imaging (MRI) has a confirmatory role, tumour show low intensity on T1 images and hyperintensity on T2, also vessels encasement is seen in a lesion with malignant potential. MRI may be useful in differentiation of pancreatic schwannoma from adenocarcinoma as schwannoma typical hyperintense pattern on T2 imaging. [7] Biopsy in the form of Endoscopic Ultrasound guided Fine Needle Aspiration (EUS-FNA) has been quite beneficial as it provides preoperative diagnosis.[8] Though diagnosis is only confirmed with post operative histopathological analysis of specimen. Typical histological pattern of schwannomas includes two areas known as Antoni A and Antoni B in different combinations. The Antoni A area is hypercellular and formed by closely packed spindle cells arranged as palisade with Verocay bodies, whereas the Antoni B area is hypocellular with loosely arranged cells. Degeneration as calcification or bleed and cystic changes are usually seen in the Antoni B area. [9,10] Further immunohistochemistry staining shows positivity for S-100 protein, vimentin, Neural Cell Adhesion Molecule (NCAM) and negative for pancytokeratin agents as AE1/AE3, desmin, SMA, CD34+ and C-kit [11].

As Tumour biology of pancreatic schwannomas is similar to neurogenic schwannomas, which is radiation amenable. So these tumours are presumed to be radiosensitive but exact role of radiation is not known till date. As the malignant transformation of pancreatic schwannomas is very rare, usually simple enucleation is usually suffice provided preoperative diagnosis confirmed with biopsy. [12] Otherwise, oncological resection in form of pancreaticoduodenectomy is indicated and recurrences are rare though close follow up is recommended.

\section{Conclusion}

Pancreatic schwannoma seems to be important differential diagnosis of pancreatic lesions. These tumors commonly have non specific symptoms but can also present with features of gastric outlet obstruction. Pre operative diagnosis is difficult while CECT/ MRI abdomen may provide some insight. Newer modality as EUS guided FNAC is promising. Though tumor resection and histopathological analysis is confirmatory. For benign tumors, simple enucleation is usually adequate, whereas malignant tumors require standard oncological resection.

\section{Competing Interests}

None.

\section{Funding}

None.

\section{Ethical Approval}

Written informed consent to publication was obtained from the patient or next of kin.

\section{Acknowledgements}

The authors thank the patient for allowing them to publish the case report and use the images taken during her stay in hospital.

\section{References}

[1] Almo KM, Traverso LW. Pancreatic schwannoma: an uncommon but important entity. J Gastrointest Surg 2001; 5: 359-363.

[2] Gupta A, Subhas G, Mittal VK, Jacobs MJ. Pancreatic schwannoma: literature review. J Surg Educ 2009; 66: 168-173.

[3] Suzuki S, Kaji S, Koike N, Harada N, Hayashi T, Suzuki M, Hanyu F, Ban S. Pancreatic schwannoma: a case report and literature review with special reference to imaging features. JOP 2010; 11: 31-35.

[4] Mummadi RR, Nealon WH, Artifon EL, Fleming JB, Bhutani MS. Pancreatic Schwannoma presenting as a cystic lesion. Gastrointest Endosc 2009; 69: 341; discussio 341.

[5] Okuma Tofigh AM, Hashemi M, Honar BN, Solhjoo F. Rare presentation of pancreatic schwannoma: a case report. J Med Case Reports 2008; 2: 268.

[6] Yu RS, Sun JZ. Pancreatic schwannoma: CT findings. Abdom Imaging 2006; 31: 103-105.

[7] Novellas S, Chevallier P, Saint Paul MC, Gugenheim J, Bruneton JN. MRI features of a pancreatic schwannoma. Clin Imaging 2005; 29: 434-436.

[8] Li S, Ai SZ, Owens C, Kulesza P. Intrapancreatic schwannoma diagnosed by endoscopic ultrasound-guided fine-needle aspiration cytology. Diagn Cytopathol 2009; 37: 132-135.

[9] Tafe LJ, Suriawinata AA. Cystic pancreatic schwannoma in a 46year-old man. Ann Diagn Pathol 2008; 12: 296-300.

[10] Eggermont A, Vuzevski V, Huisman M, De Jong K, Jeekel J. Solitary malignant schwannoma of the pancreas: report of a case and ultrastructural examination. J Surg Oncol 1987; 36: 21-25. 
[11] Weiss SW, Langloss JM, Enzinger FM. Value of S-100 protein in the diagnosis of soft tissue tumors with particular reference to benign and malignant Schwann cell tumors. Lab Invest 1983; 49: 299-308.
[12] Paranjape C, Johnson SR, Khwaja K, Goldman H, Kruskal JB, Hanto DW. Clinical characteristics, treatment, and out- come of pancreatic Schwannomas. J Gastrointest Surg 2004; 8: 706-712. 